\title{
Les Difficultés Des Apprenants Marocains À Mobiliser Les Facteurs Temps Et Espace En Géologie : Cas Des Fossiles
}

\author{
Imane Chmanti-Houari \\ Mohammed Oudrhiri Hassani \\ Laboratoire de Didactique, d’Innovation Pédagogique et Curriculaire \\ (LADIPEC), Faculté des sciences Dhar Mahraz. Fès, \\ Université Sidi Mohammed Ben Abdellah, Fès, Maroc

\section{Hassan Lachkhem} \\ Laboratoire de Géosystèmes et Développement Durable (GDD), \\ Faculté des sciences Dhar Mahraz. Fès, \\ Université Sidi Mohammed Ben Abdellah. Fès, Maroc
}

doi: 10.19044/esj.2017.v13n13p303 URL:http://dx.doi.org/10.19044/esj.2017.v13n13p303

\begin{abstract}
This work aims at diagnosing the Moroccan high school students' difficulties in perceiving and mobilizing the time and space parameters, particularly through the study of the concepts of fossils. To attain our objective, first, an analysis was carried out on Life and Earth Science programs in Moroccan high school. Then, the results achieved by the Nominal Group Technique (NGT) have enabled us to elaborate a questionnaire related to the concept of fossils. These investigation tools affirm that the students have difficulties in assimilating and mobilizing the time and space parameters, particularly while overvaluing the time factor at the expense of the space one, a confusion in the mobilization of the temporal scales and inability to imagine the succession in time and space of the different stages of the fossilization. To reduce the difficulties faced by the learners, we suggest some pedagogical solutions.
\end{abstract}

Keywords: Geology, Fossil, Space, Time, Nominal Group Technique

Résumé

Ce travail a pour objectif de contribuer à diagnostiquer les difficultés des lycéens marocains à percevoir et à mobiliser les paramètres temps et espace en géologie, notamment à travers l'étude du concept fossile. Nous avons effectué une analyse des programmes des Sciences de la Vie et de la Terre (SVT) au secondaire marocain. Puis, en se basant sur la Technique de 
Groupe Nominal (TGN), nous avons élaboré un questionnaire relatif au concept de fossile. Ces investigations confirment que nos lycéens marocains éprouvent des difficultés d'assimilation et de mobilisation des paramètres temps et espace, particulièrement une survalorisation du facteur temps en dépit du facteur espace, une confusion dans la mobilisation des différentes échelles temporelles ainsi que l'incapacité d'imaginer la succession dans le temps et dans l'espace des différentes étapes de la fossilisation. Pour atténuer les difficultés rencontrées par ces élèves, nous proposons des suggestions pédagogiques.

Mots clés: Géologie, Fossile, Espace, Temps, Technique de Groupe Nominal

\section{Introduction et problématique}

L'espace et le temps constituent deux concepts clés dans l'enseignement des Sciences de la Terre. Cependant, de nombreux travaux en didactique des sciences soulignent les difficultés des apprenants à la perception et à la mobilisation de ces deux paramètres lors de l'apprentissage des phénomènes géologiques. En effet, selon Skibine (1995: cité par Boughanmi, 2009) ces concepts sont difficiles à faire naître et à utiliser par l'apprenant. Pour Savaton (1998) les étudiants présentent des faiblesses de spatialisation. En effet, les roches ne sont pas imaginées dans l'espace, elles ne se prolongent pas dans le non vu. Enfin pour Lamarti et al. (2009), il s'agit des difficultés à assimiler les différentes échelles impliquées, à s'orienter dans l'espace, à passer des représentations bidimensionnelles à des représentations tridimensionnelles ou à changer de référentiel d'observation.

Jugée difficile, la géologie est pourtant présente dans les programmes secondaires. Les travaux qui se sont intéressés à son apprentissage (Chalak, 2013 ; Lamarti, 2009) sont cependant peu nombreux par rapport à ceux rapportés dans la littérature pour l'enseignement d'autres disciplines expérimentales.

\section{Cadre théorique}

Nous donnons tout d'abord un aperçu sur les difficultés de mobilisation des facteurs temps et espace lors de l'apprentissage de quelques phénomènes géologiques. Ensuite, nous relatons des obstacles épistémologiques liés à l'assimilation du concept fossile. Enfin, nous présentons une description de l'enseignement des Sciences de la Terre (ST) dans les programmes secondaires marocains suivi de l'objectif et l'hypothèse de la recherche. 


\section{Difficultés de mobilisation des facteurs temps et espace lors de l’apprentissage de quelques phénomènes géologiques}

De nombreux travaux en didactique des sciences de la terre ont été consacrés aux difficultés de mobilisation des facteurs temps et/ou espace, que rencontrent les apprenants en géologie.

Dans ce cadre, la théorie de la tectonique des plaques constitue un modèle de la mécanique terrestre qui a un pouvoir unificateur des grands phénomènes géologiques. Néanmoins, plusieurs travaux affirment que les apprenants ne conçoivent pas l'intérêt de ce modèle. En France, Monchamp et Skibine (1995) renvoient les difficultés d'assimilation de la théorie de la tectonique des plaques par les élèves du primaire et du secondaire aux différences d'échelles et aux concepts difficiles impliqués : temps, espace, viscosité.

De même, Orange (2003) a démontré que les lycéens français évoquent des difficultés quant à la mobilisation de l'espace et du temps lors de l'explication du phénomène de l'extension du fond océanique à l'origine de la tectonique des plaques. En effet, l'accrétion et la sédimentation constituent deux processus instantanés, mais ne se produisent pas exactement dans la même direction spatiale. Enfin, Boughanmi (2009) a souligné que les explications catastrophistes et fixistes évoquées par les apprenants tunisiens à propos des phénomènes géologiques impliqués dans la tectonique des plaques, tels que les séismes, le volcanisme et l'orogenèse, empêchent leur conception de l'immensité du temps impliqué.

En outre, le volcanisme est un phénomène géologique qui semble être relativement accessible à l'observation directe, grâce aux médias, et ses manifestations peuvent être étudiées à l'échelle Humaine dans le temps et dans l'espace. Cependant, Tacussel (1995) a remarqué que l'assimilation des processus à l'origine de ce phénomène est problématique pour les élèves français au primaire. En effet, les éruptions volcaniques constituent des manifestations de courte durée, à l'échelle Humaine, mais résultent en réalité d'un ensemble de phénomènes de longue durée à l'échelle du temps géologique. En fait, les élèves trouvent des difficultés à raccorder des échelles de temps totalement différentes.

Par ailleurs, les crises biologiques sont des discontinuités majeures à l'échelle planétaire dans l'échelle du temps géologique et qui séparent des périodes de plus grande stabilité. Par exemple, parmi les grandes coupures référencées dans l’échelle stratigraphique internationale, la crise biologique dite du Crétacé/Tertiaire a entraîné la disparition d'une quantité massive d’organismes, notamment tous les dinosaures, de nombreux invertébrés marins ainsi que des végétaux. Cette extinction globale et relativement brutale qui a affecté l'ensemble de la planète s'est produite sur une courte période de temps à l'échelle géologique. Les résultats des études de 
Boughanmi (2009) ont, cependant, démontré que les lycéens tunisiens comprennent la brutalité de cette extinction comme une période courte à l'échelle Humaine car ils l'assimilent à des catastrophes naturelles actuelles telles qu'un séisme ou un tsunami qui ne se déroulent que sur de très courtes durées. Or, il a fallu au moins quelques millions d'années pour qu'une telle extinction se produise. Cet auteur affirme que l'influence du catastrophisme actuel sur les explications des élèves constitue un obstacle à leur conception de l’immensité du temps géologique.

\section{Obstacles épistémologiques liés au concept fossile}

Étymologiquement, le mot fossile vient du latin fossilis et désigne tout ce qui est tiré de la terre, aussi bien les roches et minerais que les animaux pétrifiés ; progressivement ce terme ne s'est plus appliqué seulement qu'aux restes d'êtres vivants, selon Gouanelle et Schneeberger (1995). Si on considère la définition de l'encyclopédie des fossiles (Edition atlas, 1987), ces derniers constituent tous les organismes dont des parties ont été conservées soit par pétrification ou par fossilisation mais aussi les traces, ou ichnofossiles, qu'ils ont laissées de leurs corps, de leurs déplacements et de leurs activités.

Les fossiles sont des marqueurs précis de temps, sur une échelle stratigraphique, par chronologie relative; ce sont aussi des indicateurs de faciès des paléo environnements; et enfin, ils constituent des témoins en faveur de la théorie synthétique de l'évolution des espèces.

Certains travaux en didactique des sciences de la terre ont abordé les diverses difficultés rencontrées par les élèves lors de la construction du concept fossile. Astolfi et Peterfalvi (1993 : cités par Tacussel, 2002) ont souligné que les concepts de fossile et de fossilisation constituent le noyau convergent de plusieurs obstacles épistémologiques liés à la construction des concepts de temps, d'espace et de transformation de la matière. En particulier, Gouanelle et Schneeberger (1995) ainsi que Tacussel (2002) ont montré que les élèves français au primaire considèrent les fossiles comme des constructions humaines en raison d'un obstacle artificialiste. Ils ont aussi signalé la forte présence des conceptions fixistes : pour ces enfants la roche encaissante est immuable et préexiste, l'organisme s'imprimant en surface, ou y rentrant en force, ou par l'effet de la durée. À ces difficultés s'ajoutent celles liées à la perception des durées et de la chronologie.

À la lumière de ces travaux, on a jugé intéressant de vérifier si les lycéens marocains évoquent les mêmes types de difficultés. 


\section{L'enseignement des Sciences de la Terre dans les programmes secondaires marocains}

Dans les programmes secondaires marocains, l'enseignement de la géologie ou Sciences de la Terre (ST) fait partie des programmes des Sciences de la Vie et de la Terre (SVT).

Au collège, l'enseignement de la géologie est limité à la première et à la deuxième année. En première année, pour les élèves de 12 à 13 ans, les cours de géologie portent sur la géodynamique externe: genèse et classification des roches sédimentaires, notions de fossile et fossilisation et initiation à la notion d'échelle bio-stratigraphique. En deuxième année, ces cours se focalisent sur la géodynamique interne : la dérive des continents, la notion de plaques lithosphériques, le volcanisme, les séismes et les différents types des chaînes de montagne dans leur contexte tectonique ainsi que la mise en évidence de la structure interne de la terre à partir des études sismiques.

$\mathrm{Au}$ secondaire qualifiant, les chapitres consacrés à la géologie se répartissent sur les trois années composant ce cycle : un tronc commun (TC), la première année baccalauréat $\left(1^{\mathrm{re}} \mathrm{BAC}\right)$ et la deuxième année baccalauréat $\left(2^{\mathrm{e}} \mathrm{BAC}\right)$. Ces cours de géologie comprennent : en tronc commun, l'étude des composants des écosystèmes et leurs interactions ainsi que des notions de géodynamique externe; en $1^{\text {re }}$ année BAC, la reconstitution de l'histoire géologique des paléo-environnements (notion de biozone, notions de fossiles de faciès et de fossiles stratigraphiques et intérêt des fossiles pour la construction de l'échelle bio-stratigraphique) et enfin en $2^{\mathrm{e}}$ année BAC, élèves de 17 à 18 ans, les programmes portent sur l'étude de la formation des chaînes des montagnes dans leurs contextes tectoniques.

Tableau 1

Volumes horaires des Sciences de la Terre (ST) et des Sciences de la Vie (SV) au collège et au secondaire qualifiant

Au collège Au secondaire qualifiant

\begin{tabular}{ccccccc}
\cline { 2 - 7 } Volume horaire & $1^{\text {re }}$ année & $2^{\mathrm{e}}$ année & $3^{\mathrm{e}}$ année & $1^{\text {re }}$ année & $2^{\mathrm{e}}$ année & $3^{\mathrm{e}}$ année \\
\hline S.T & 28 & 28 & 0 & 51 & 34 & 34 \\
SV & 28 & 28 & 56 & 51 & 102 & 170 \\
\hline SVT & 56 & 56 & 56 & 102 & 136 & 204 \\
\hline
\end{tabular}

Par ailleurs, l'examen du tableau $n^{\circ} 1$, montre que le volume horaire imparti aux Sciences de la Vie est nettement plus important que celui consacré aux Sciences de la Terre. Ce volume horaire réduit n'est certainement pas sans affecter négativement l'attrait de cette discipline auprès des élèves et même pour quelques enseignants. À noter que c'est le même enseignant qui assure les cours de la biologie et de la géologie. De plus, en raison du volume horaire restreint et du programme que nous 
estimons relativement long, beaucoup d'enseignants des SVT se trouvent contraints d'annuler les sorties géologiques ainsi que certaines séances de travaux pratiques, pourtant essentiels pour l'assimilation et la compréhension de la géologie.

Enfin, le positionnement des phénomènes géologiques dans le temps et dans l'espace constitue un des principaux objectifs visés par les programmes de géologie dans le secondaire marocain.

Dans ce sens et pour évaluer la capacité des lycéens marocains à mobiliser les facteurs temps et espace, nous avons mené une étude sur leur conception et leur assimilation des notions de fossile et de fossilisation. En effet, la fossilisation, c'est-à-dire la transformation d'un organisme mort en fossile est un phénomène qui se réalise en plusieurs étapes se succédant dans le temps et dans l'espace : mort et dépôt de l'organisme, surplace ou après transport; ensevelissement du cadavre; dissolution des parties molles et enfin minéralisation ou formation de moules internes ou externes selon les propriétés chimiques des parties dures et du sédiment encaissant.

\section{Objectif de la recherche}

La présente recherche entreprend de dévoiler les difficultés que les lycéens marocains rencontrent vis-à-vis de la mobilisation des facteurs temps et espace lors de l'étude des notions de fossile et de fossilisation présents dans le programme du secondaire qualifiant.

\section{Hypothèse de la recherche}

Les difficultés de mobilisation d'espace et de temps entravent la capacité des lycéens à assimiler le concept fossile et à retracer son histoire évolutive dans le temps et l'espace.

\section{Méthodologie}

Pour atteindre notre objectif, on s'est basé sur la technique de groupe nominal dont les résultats nous ont permis l'élaboration d'un questionnaire.

\section{Participants}

Durant les deux épisodes de notre investigation, technique de groupe nominal et questionnaire, nous avons travaillé avec des lycéens de première année baccalauréat, option sciences expérimentales, âgés de 16 à 17 ans, appartenant à deux établissements du secondaire qualifiant, du secteur public, de l'Académie Régionale de l'Éducation et de la Formation Fès Boulmane : lycée Moulay Rachid et lycée Omo Ayman. Les deux établissements sont situés dans un milieu urbain de la ville de Fès.

Notre étude est qualitative. Pour que les résultats observés soient généralisables à l'ensemble des lycéens marocains, la procédure de choix des participants est celle de 
l'échantillonnage aléatoire où les membres de la population ont la même probabilité de faire partie de l'échantillon, les élèves questionnés suivent, en effet, le même programme et dans les mêmes conditions que leurs collègues dans les autres lycées marocains.

Tout d'abord, nous avons réalisé la technique de groupe nominal sur 15 lycéens du lycée Moulay Rachid le 03/02/14. Les participants à la technique de groupe nominal se sont présentés volontairement à la suite d'une affiche publiée dans leur établissement une semaine avant le déroulement de l'étude. Ensuite, en se référant aux résultats de cette technique nous avons établi un questionnaire qu'on a soumis à un échantillon de 135 lycéens appartenant aux deux établissements concernés.

\section{Recueil de données}

\section{Technique de groupe nominal}

Cette technique a été établie par Delbecq et Van de Ven (1971). C'est le fruit des études psychologiques de fonctionnement de petit groupe de 10 à 15 personnes. Ses principaux avantages consistent à permettre à chacun des membres d'un groupe de discuter, de s'exprimer librement sur le sujet, d'intervenir dans le débat autant que les autres participants et de faire valoir, par vote secret, son opinion personnelle sur les idées émises tout en évitant les problèmes habituels reliés aux groupes de discussion.

Cette méthode consiste à présenter au groupe une question ouverte appelée la question nominale. Celle-ci doit être claire, précise et univoque (Grenier et Lagarde, 2000). Elle permet aux membres du groupe, dans un laps de temps relativement court, de produire une liste importante de suggestions qui devraient être examinées et classées, par la suite, par ordre de priorité, selon le vote des membres du groupe.

\section{Enquête par questionnaire}

Sur la base des résultats de notre technique de groupe nominal, nous avons élaboré un questionnaire composé de trois questions qui sont de type : ouverte, c'est-à-dire sans aucune proposition de réponses; fermée, c'est-à-dire avec proposition d'un choix multiple de réponses; et semi-ouverte, c'est à dire composée de deux sous-questions, l'une ouverte et l'autre fermée.

La première question vise à comprendre comment les élèves identifient le fossile, la deuxième se focalise sur l'intérêt des fossiles alors que la troisième question cherche à élucider comment les élèves conçoivent la relation fossile-encaissant ou déroulement du phénomène de fossilisation.

\section{Déroulement}

Notre recherche s'est déroulée pour les deux lycées au début du deuxième semestre. Le nombre des participants et les dates du déroulement 
du questionnaire au sein des deux établissements sont pour le lycée Moulay Rachid 75 élèves le 17/02/14 et pour le lycée Omo Ayman 60 élèves. le 04/03/14.

$\mathrm{Au}$ cours du déroulement de la technique de groupe nominal, nous avons posé, dans la première séance, qui a duré une heure, la question nominale $\mathrm{n}^{\circ} 1$. Après avoir expliqué l’objectif de notre question, nous avons encouragé les élèves à s'exprimer librement. Après une semaine, le même groupe est réuni, dans la même salle et dans les mêmes conditions, puis nous avons posé la deuxième question nominale.

Après traitement et analyse des résultats de la technique de groupe nominal, nous avons élaboré puis soumis notre questionnaire anonyme aux 135 participants. Nous avons ensuite procédé par l'analyse de contenu thématique catégorielle (Landry, 1993) comme méthode de traitement des réponses aux questions ouvertes et semi-ouvertes : elle consiste à analyser les textes ou les transcriptions des élèves puis à regrouper en catégories tous les énoncés qui se rejoignent par le sens. Le dépouillement et l'analyse des données quantifiables de la question fermée sont réalisés à l'aide du logiciel, sphinx 0.4 , relatif au traitement de données statistiques. Les résultats ci-après sont construits sur 135 observations correspondant au nombre total des participants. Les pourcentages représentent le nombre de fois qu'une réponse a été citée par les participants par rapport au total des citations.

\section{Considérations éthiques}

Notre enquête a été effectuée suite à une autorisation fournie par la Délégation du Ministère de l'Éducation Nationale de la ville de Fès. Ensuite, après explication de l'objectif de l'étude et lecture attentive du contenu de l'enquête, les directeurs des lycées ciblés ont autorisé le déroulement de l'enquête au sein de leurs établissements. À leur tour les enseignants responsables des cours des sciences de la vie et de la terre ont informé leurs élèves de l'objectif de l'enquête et de son caractère non obligatoire en invitant les volontaires à s'inscrire auprès de l'administration de leurs établissements. Pour assurer l'anonymat des réponses, les participants à l'enquête ont été invités à ne pas mentionner leurs identités sur les copies. Enfin, nous avons avisé les enseignants et les responsables administratifs des établissements concernés qu'ils recevront les résultats de l'enquête, sous forme de tiré à part, dès la publication de notre travail.

\section{Résultats et analyses}

\section{Analyse des résultats de la technique de groupe nominal}

Les résultats de cette technique ont permis de mettre en évidence des catégories de conceptions en rapport avec le concept fossile. 


\section{Question nominale $\mathbf{n}^{\circ} \mathbf{1}$ de la technique de groupe nominal}

Les réponses à cette question, qui demande aux apprenants de citer une liste de mots et/ou d'expressions en rapport avec le mot fossile, sont classées par ordre d’importance décroissant dans le tableau $n^{\circ} 2$. La valeur 9 correspond à la réponse la plus citée jusqu’à la valeur 1 pour la moins citée.

\section{Tableau 2}

Réponses à la question nominale $\mathrm{n}^{\circ} 1$, pour citer une liste de mots ou d'expressions en rapport avec le mot fossile, classées par ordre d'importance décroissant

\begin{tabular}{cccc}
\hline Réponses & Poids & Ordre & $\begin{array}{c}\text { Nombre de } \\
\text { citations }\end{array}$ \\
\hline Fossiles stratigraphiques & 104 & 1 & 13 \\
Fossiles de faciès & 97 & 2 & 12 \\
Trilobite, ammonite, nummulites, dents de requins & 76 & 3 & 11 \\
Restes et débris d'animaux anciens & 72 & 4 & 8 \\
Traces de déplacement des dinosaures, traces & 69 & 5 & 7 \\
d'activités d'organismes vivants & & & \\
Organismes microscopiques & 64 & 6 & 6 \\
Formés grâce à la nature & 39 & 7 & 4 \\
Se forment dans les roches sédimentaires & 37 & 8 & 4 \\
Organismes marins & 35 & 9 & 5 \\
Eau & 34 & 10 & 4 \\
Archéologie & 31 & 11 & 3 \\
Paléogéographie & 28 & 12 & 3 \\
Apparaissent grâce à l'érosion & 23 & 13 & 3 \\
\hline
\end{tabular}

\section{Ces réponses ont permis de distinguer entre quatre catégories de conceptions}

La première catégorie regroupe la majorité des élèves par 30 citations, soit $38 \%$ du nombre total de citations qui évoquent des conceptions faisant allusion à l'origine du fossile par : des restes et débris d'animaux anciens ; organismes microscopiques; traces de déplacement de dinosaures; traces d'activités d'organismes vivants et/ou à son milieu de vie comme: organismes marins.

La deuxième catégorie, est formée de 27 citations, soit 34,2\%, renvoyant à l'intérêt des fossiles en tant qu'indicateurs spatio-temporels : des fossiles de faciès et/ou fossiles stratigraphiques ou paléogéographie.

La troisième catégorie rassemble 11 citations, soit 13,9\% faisant allusion aux conditions de fossilisation : les fossiles se forment grâce à la nature ; apparaissent grâce à l'érosion; se trouvent dans les couches sédimentaires.

La dernière catégorie englobe 11 citations, soit 13,9 \% rappelant des exemples de fossiles déjà confrontés dans le cours ou évoqués dans les médias : les dinosaures ; les trilobites ; les ammonites ; les nummulites ou les dents de requins. 


\section{Question nominale $\mathbf{n}^{\circ} \mathbf{2}$ de la technique de groupe nominal}

Dans cette question, on a demandé aux élèves de citer les obstacles qui bloquent leur compréhension du phénomène de fossilisation.

\section{Tableau 3}

Réponses à la question nominale $n^{\circ} 2$, sur les obstacles qui bloquent la compréhension des participants du phénomène de fossilisation, classées par ordre d’importance décroissant

\begin{tabular}{cccc} 
Réponses & Poids & Ordre & $\begin{array}{c}\text { Nombre } \\
\text { de } \\
\text { citations }\end{array}$ \\
\hline $\begin{array}{c}\text { La géologie en général est une science très difficile } \\
\text { Manque de travaux pratiques }\end{array}$ & 99 & 1 & 14 \\
$\begin{array}{c}\text { ou non } \\
\text { Difficulté de distinguer s'il s'agit d'un organisme fossile }\end{array}$ & 76 & 2 & 12 \\
$\begin{array}{c}\text { Difficulté d'imaginer comment se forment les fossiles } \\
\text { Illustrations des cours par des images de fossiles et non }\end{array}$ & 76 & 4 & 6 \\
de vrais fossiles à toucher et à observer & 74 & 5 & 6 \\
$\quad$ Manque de sorties de terrain & 67 & 6 & 10 \\
Absence de motivations pédagogiques & 63 & 7 & 5 \\
Termes paléontologiques très difficiles & 51 & 8 & 9 \\
La fossilisation est un phénomène qu'on ne peut pas & 49 & 9 & 5 \\
observer directement dans la nature & & & 8 \\
$\begin{array}{c}\text { Cours trop chargés et temps alloué limité } \\
\text { scolaire) }\end{array}$ & 38 & 10 & 5 \\
Méthodes d'enseignement dépassées (tableau, manuel & 37 & 11 & 4 \\
$\begin{array}{c}\text { Communications mutuelles limitées dans la classe } \\
\text { Explications superficielles }\end{array}$ & 31 & 12 & 3 \\
\hline
\end{tabular}

D’après le tableau $n^{\circ} 3$, les réponses faisant allusion aux obstacles d'ordre pédagogique représentent les poids et les pourcentages les plus élevés avec 63 citations, soit 58,9\% du nombre total de citations. Il s'agit des difficultés en relation avec des méthodes pédagogiques en l'occurrence les cours illustrés par des images et non pas par de vrais fossiles, le manque de travaux pratiques et de sorties de terrain.

Les obstacles liés à la discipline géologie sont également cités dans des réponses avec un poids et un pourcentage relativement important soit $21,5 \%$, avec 23 citations, qui considèrent que la géologie est en général une discipline compliquée, difficile ou souvent incompréhensible comme l'exemple des termes paléontologiques.

Les obstacles spécifiques au concept fossile concernent des réponses avec un poids et un pourcentage presque aussi important que la catégorie précédente par 21 citations soit 19,6\%. Elles renvoient aux difficultés de l'identification des fossiles et de l'imagination du déroulement du phénomène de fossilisation qu'on ne peut pas observer directement dans la nature. 
Nous constatons que les difficultés exprimées par les lycéens sont liées d'une part, aux méthodes pédagogiques adoptées en classe et à la nature de la géologie en tant que discipline ardue, d'autre part à l'assimilation du concept fossile. Ceci nous a guidé à l'élaboration d'un questionnaire pour la suite de notre enquête.

\section{Analyse des résultats de l'enquête par questionnaire}

Nous rapportons ici les réponses des participants aux trois questions qui composent notre enquête ainsi que leur traitement.

\section{Question $\mathbf{n}^{\circ} 1$}

Celle-ci concerne la définition d'un fossile et des objets considérés comme fossiles, selon une liste proposée.

Tableau 4

Questions sur la définition d'un fossile et objets considérés comme fossiles

$$
\text { - Qu'est-ce qu'un fossile? }
$$

- Indiquez parmi la liste suivante les objets que vous considérez comme fossiles en mettant le signe $(\times)$ dans la case oui ou non. Essayer de justifier vos réponses

\begin{tabular}{|c|c|c|c|}
\hline Objets & Oui & Non & Explication \\
\hline \multicolumn{4}{|l|}{ Tronc d'arbre silicifié } \\
\hline \multicolumn{4}{|c|}{ Moulage externe d'oursin } \\
\hline \multicolumn{4}{|c|}{ Coquille d'Ammonite } \\
\hline \multicolumn{4}{|c|}{ Valves d’huîtres actuelles } \\
\hline \multicolumn{4}{|c|}{ Moulage interne de Trilobite } \\
\hline \multicolumn{4}{|c|}{ Bactéries conservées dans les cristaux de sel } \\
\hline \multicolumn{4}{|c|}{ Traces de déplacements de dinosaures } \\
\hline \multicolumn{4}{|c|}{ Travertins } \\
\hline Traces de terriers & & & \\
\hline
\end{tabular}

\section{Nous avons réparti les réponses en quatre catégories.}

La première catégorie englobe la majorité des réponses des élèves, avec $75,7 \%$, qui définit un fossile en se basant sur sa nature animal, végétal ou minéral avec une prédominance, par $70 \%$, des réponses renvoyant à la nature animale et seulement 5,7\% pour les autres natures, les fossiles sont des restes d'animaux ou des animaux disparus. Nous pensons que cela s'explique par l'influence des illustrations et des exemples des fossiles présentés dans les cours et par les médias. En effet, les cours de paléontologie au secondaire sont illustrés essentiellement par des exemples de fossiles animaux et rarement végétaux. De plus, les médias donnent généralement plus d'importance aux fossiles animaux comme les dinosaures ou les coquilles au détriment des fossiles végétaux.

En outre, 3,5 \% de réponses de ces élèves, assimilent les fossiles à des roches et sont donc d'origine minérale. Nous pensons que ces conceptions 
sont le résultat du raisonnement par couple notionnel (Tacussel, 2002), où deux termes sont associés, l'un appelant l'autre. Pour ces apprenants, tout ce qui est dur est une roche comme le moulage externe d'oursin est un fossile parce que c'est une roche sédimentaire ou le tronc d'arbre silicifié est un fossile parce qu'il est induré.

D’autres élèves, avec 13,7 \%, des réponses, ont évoqué, en plus de la nature d'un fossile, les modes de fossilisation en citant les empreintes, les traces de déplacement des dinosaures, les moulages, les traces de terriers ou d'activités animales.

Enfin, certains élèves, avec 3,2 \% des réponses, renvoient à une origine artificielle ; ils pensent que les fossiles sont sculptés par l'Homme.

Les réponses formant la $2^{\text {éme }}$ catégorie, avec 17,6\%, évoquent les phénomènes à l'origine de la fossilisation. Dans cette catégorie on distingue entre deux types de réponses: 14,6\% de ces réponses mobilisent les processus de fossilisation ou de sédimentation; enfouissement; érosion; dégradation de la matière organique; conservation des parties dures. Cependant, on note que ces apprenants considèrent qu'un fossile résulte d'un seul phénomène : soit la sédimentation, soit l'enfouissement ou la dégradation de la matière organique et la conservation des parties dures. Ceci dénote de la méconnaissance de la succession des différentes étapes de la fossilisation dans le temps, ou encore de leur succession dans l'espace comme dans le cas des organismes transportés après leur mort et qui se fossilisent en dehors de leurs milieux de vie. On note aussi une confusion chez certains élèves entre les processus de la sédimentation et ceux de la fossilisation. En effet, pour ces élèves les fossiles résultent de la sédimentation ; se forment à cause de la sédimentation ou sont des restes d’êtres vivants formés sous l'effet de la sédimentation.

En outre, $3 \%$ de réponses évoque des explications généralistes en se référant à l'influence de la nature et la volonté de Dieu : on peut expliquer l'existence des fossiles au sein des roches par les conditions naturelles ; Dieu facilite tous ces phénomènes; les fossiles sont des restes d'organismes qui ont subi un ensemble de processus naturels. Ceci s’explique par la difficulté d’imagination des phénomènes non constatés.

La catégorie 3, avec 4,7\%, rassemble les réponses évoquant seulement le facteur temps sans aucune allusion au facteur espace. Différentes facettes de ce facteur temps sont signalées soit par l'ancienneté des fossiles en citant l'échelle temporelle: c'est au cours du temps géologique que les fossiles peuvent se former au sein des roches; tout organisme peut se transformer au cours du temps géologique en un fossile ou sans préciser l'échelle temporelle en déclarant que des organismes anciens ont vécu dans le passé. D’autres réponses rappellent l'utilisation des fossiles pour la datation relative en pensant qu'ils servent à la datation des roches 
sédimentaires ; les fossiles peuvent nous renseigner sur l'âge des roches. Le reste des réponses considère le temps comme seul facteur à l'origine de la formation des fossiles : grâce au temps.

La $4^{\text {éme }}$ et dernière catégorie est une minorité d'apprenants avec $2 \%$ qui a mobilisé des conceptions renvoyant seulement au facteur espace soit en termes de localisation des fossiles; les fossiles sont des objets qui se trouvent dans le sous-sol, à l'intérieur des strates, dans les roches, dans les bassins sédimentaires ou dans le milieu marin ou bien en termes de transport : les fossiles se transportent d'un milieu à un autre, puis ils se sédimentent.

Le nombre de citations et le pourcentage des réponses par catégorie sont regroupés dans le tableau $n^{\circ} 5$ ci-dessous :

Tableau 5

Nombre de réponses et pourcentage par catégorie à la question nominal $\mathrm{n}^{\circ} 1$

\begin{tabular}{ccc}
\hline Catégories & Nombres de citations & Pourcentage des réponses \\
\hline Catégorie 1 & 224 & 75,7 \\
Catégorie 2 & 52 & 17,6 \\
Catégorie 3 & 14 & 4,7 \\
Catégorie 4 & 6 & 2 \\
\hline Total & 296 & 100 \\
\hline
\end{tabular}

En bref, nous constatons que les définitions d'un fossile transcrit par nos lycéens mobilisent rarement le facteur temps avec $4,7 \%$. On a remarqué en outre une sous-estimation du facteur espace qui est quasiment absent avec $2 \%$.

\section{Question $\mathbf{n}^{\circ} 2$}

L'objectif de la question $n^{\circ} 2$ est de révéler l'assimilation par les participants de l'intérêt des fossiles comme indicateurs spatio-temporels par des fossiles de faciès et/ou fossiles stratigraphiques. Ces conceptions sont présentées sur le tableau $n^{\circ} 6$ ci-dessous.

Tableau 6

Conception selon les élèves, des intérêts des fossiles comme indicateurs spatio-temporels

Quel sont les intérêts des fossiles ?

La reconstitution du paléo-environnement

La reconstitution de la paléogéographie

La reconstitution des étapes de l'évolution de la vie sur terre

La datation des roches

La détermination de la structure interne de la terre

Nous avons réparti les réponses à cette question en trois catégories : la première regroupe les propositions renvoyant aux deux types d'intérêt, temporel et spatial avec 50,5\%, la deuxième avec 28,3\% sélectionne les propositions renvoyant aux fossiles comme indicateurs temporels seulement 
en citant la datation des roches, la structuration et le découpage de l'échelle du temps géologique ou la reconstitution des étapes de l'évolution de la vie sur terre. Enfin la troisième catégorie avec 21,2 \% correspond aux propositions considérant les fossiles comme indicateur spatial seulement soit par la reconstitution du paléo-environnement ou la reconstitution de la paléogéographie.

Les réponses montrent un bon niveau de connaissance des intérêts des fossiles et vont dans le sens de celles obtenues par la technique de groupe nominal, où 34,2 \% des lycéens ont évoqué les intérêts des fossiles en termes de fossiles de faciès et/ou de fossiles stratigraphiques.

Cependant, les résultats obtenus montrent que presque la moitié des réponses avec 49,5 \% sous-estime l'intérêt à la fois temporel et spatial des fossiles et leur attribue soit seulement un intérêt temporel avec 28,3\%, soit seulement un intérêt spatial avec 21,2 \%. Ceci peut bloquer la reconstruction d'un modèle explicatif clair de l'évolution des mécanismes de fossilisation dans le temps et dans l'espace.

\section{Question $\mathbf{n}^{\circ} 3$}

La question $n^{\circ} 3$ qui demande aux élèves d'expliquer comment un fossile peut-il se retrouver dans une roche, a pour objectif principal l'évaluation de leur conception sur les différentes étapes de la fossilisation, en particulier la relation fossile-encaissant.

On note en premier lieu qu'une partie considérable des apprenants avec 26,4 \% n’a pas donné de réponse. Ces résultats sont déjà constatés par la technique de groupe nominal, où $19,6 \%$ des lycéens éprouvent des difficultés d'imagination des processus mis en jeu dans la fossilisation à savoir la mort ou le dépôt des organismes, l'enfouissement in situ ou après le transport ou la diagenèse. Ceci peut s'expliquer par la non assimilation des différentes étapes de la fossilisation qui pourtant faisant partie du programme du secondaire collégial.

Les réponses de 73,5 \% des élèves sont répertoriées en 4 catégories sur le tableau $n^{\circ} 7$ :

Tableau 7

Nombre de réponses et pourcentage par catégorie à la question $n^{\circ} 3$

\begin{tabular}{ccc}
\hline Catégories & Nombre de citations & Pourcentage des réponses \\
\hline Sans réponse & 52 & 26,4 \\
Catégorie 1 & 67 & 34 \\
Catégorie 2 & 54 & 27,4 \\
Catégorie 3 & 18 & 9,1 \\
Catégorie 4 & 6 & 3 \\
\hline Total & 197 & 99,9 \\
\hline
\end{tabular}

La catégorie 1 avec $34 \%$ regroupe les réponses évoquant les conditions naturelles sans toutefois préciser comment un fossile peut se 
retrouver dans une roche. En particulier, 12,7 \% de l’ensemble des réponses des élèves appartenant à cette catégorie 1 rappellent le facteur érosion en expliquant l'existence d'un fossile au sein de la roche par la dégradation de cette dernière sous l'effet des facteurs climatiques ; les fossiles apparaissent après l'érosion ou à cause de l'érosion. Ces réponses ne font aucune allusion aux différentes étapes de la fossilisation. Ceci témoigne là aussi, soit d'une méconnaissance totale des principales étapes de la fossilisation qui se déroulent dans le temps et dans l'espace soit de l'ignorance de toute l'histoire anté-érosion.

La $2^{\text {éme }}$ catégorie avec $27,4 \%$ rassemble les réponses rappelant seulement certains processus de la fossilisation en l'occurrence la sédimentation et/ou l'enfouissement : soit à cause de la sédimentation soit les organismes s'enfouissent au sein des sédiments après leur mort.

Les réponses mobilisant le facteur temps avec 9,1\% constituent la $3^{\text {éme }}$ catégorie, soit en faisant allusion à quelques étapes de la fossilisation et leur succession dans le temps, mais sans préciser l'échelle temporelle, par des expressions comme depuis longtemps; des organismes après leur mort se sont enfouis au sein des sédiments, puis ils ont subi des transformations, et enfin le tout se transforme en roches. Elles peuvent, par ailleurs mobiliser l'échelle Humaine en tant qu'échelle de temps mais sans préciser les processus de fossilisation: après plusieurs générations les fossiles animaux et végétaux se forment dans les roches ou bien il faut des siècles pour qu'un fossile se forme dans une roche. Enfin, d’autres réponses considèrent le facteur temps suffisant pour l'incorporation des fossiles dans les roches, sans préciser ni l'échelle temporelle ni les étapes de la fossilisation : par grâce au temps.

La $4^{\text {éme }}$ catégorie correspond à une minorité de réponses avec $3 \%$ qui considère que les fossiles sont des animaux qui se sont fossilisés en "entrant, en "se cachant ou en "s'abritant" dans les roches préexistantes. Cette catégorie évoque le facteur espace par la relation entre le fossile et son encaissant. De plus, ces conceptions supposent la non-contemporanéité entre fossile et roche encaissante : les animaux s'abritent dans les trous des roches après leur mort, ils s'emprisonnent dedans ; les organismes entrent et se sédimentent dans les roches préexistantes. Ce type de réponse témoigne encore une fois d'une méconnaissance totale des différentes étapes de la fossilisation et de leur déroulement dans le temps et dans l'espace.

En conclusion, le point commun entre la quasi-totalité des réponses est l'ambigüité dans l'imagination de l'évolution spatio-temporelle. En effet, les réponses évoquant ces processus peuvent être réparties entre celles ne faisant aucune allusion au facteur temps, ni échelle ni succession dans le temps des phénomènes impliqués et celles évoquant la succession de quelques étapes de 
la fossilisation, en citant quelques facettes de temps sans aucune allusion au facteur espace.

\section{Discussion}

La plupart des travaux liés aux difficultés d’assimilation des concepts géologiques se sont penchés principalement sur les difficultés de mobilisation par les apprenants du facteur temps (Boughanmi, 2009 ; Orange, 2003 ; Skibine, 1995). Seuls quelques travaux se sont intéressés à la notion temps-espace (Gohau, 1990, 1995; Savaton, 1998); pourtant, le temps et l'espace sont deux paramètres intervenant simultanément pour tout phénomène géologique. Dans ce sens, notre étude a visé l’évaluation de la capacité des lycéens marocains à mobiliser ces deux facteurs à travers l'assimilation du concept fossile.

Pour atteindre notre objectif, nous avons adopté deux méthodes d'analyse: la technique de groupe nominal et un sondage par un questionnaire. Cette méthodologie nous a permis de pointer quelques difficultés éprouvées par nos apprenants.

La technique de groupe nominal a montré que ces lycéens trouvent l'étude des fossiles ardue à cause des difficultés de leur identification et de l'imagination des processus mis en jeux lors de leur formation. Ils justifient cela, d'une part, par le caractère de la discipline géologie jugée incompréhensible et complexe et d'autre part, par le manque voire même l'absence de certaines activités pédagogiques, en particulier les sorties sur le terrain et les travaux pratiques.

Les résultats du questionnaire montrent, quant à eux, que la majorité avec $75,7 \%$ des lycéens ciblés, dans leurs réponses à la question $\mathrm{n}^{\circ} 1$, attribue aux fossiles soit une origine animale sans évoquer la possibilité de l'origine végétale soit une origine minérale en les assimilant à des roches, enfin une origine artificielle en déclarant que les fossiles sont sculptés par l'Homme. Ces conceptions corroborent avec celles signalées dans des travaux antérieurs, notamment ceux de Gouanelle et Schneeberger (1995) et Tacussel (2002). Par ailleurs, pour 17,6 \% de ces élèves, les fossiles résultent d'un seul phénomène géologique qui est soit l'enfouissement, soit la sédimentation ou soit la dégradation de la matière organique et la conservation des parties dures ; sans citer les autres étapes de la fossilisation qui devront être mentionnées.

De plus, nous constatons que dans leurs définitions, les participants éprouvent des difficultés de mobilisation des facteurs temps et espace. En effet, seulement 4,7\% des réponses évoquent le facteur temps et cela selon trois facettes : en terme d'ancienneté des fossiles sans, cependant, préciser l'échelle temporelle; en citant les fossiles en tant qu'outils de datation relative ou en considérant le temps comme facteur suffisant à la formation 
d'un fossile. Enfin, on note que les définitions évoquant le facteur espace sont très rares soit $2 \%$ de l'ensemble des réponses, et qu'elles se focalisent sur la localisation spatiale des fossiles ou sur la possibilité de leur transport.

Nos lycéens semblent cependant, plus facilement mobiliser ces facteurs temps et espace lors de leurs réponses à la question $n^{\circ} 2$ relative aux intérêts des fossiles. En effet, puisque la moitié d'entre eux, 50,5 \%, insiste sur leurs intérêts spatio-temporels: datation des roches; reconstitution des paléoenvironnements ; reconstitution de la paléogéographie. L’autre moitié leur attribue, soit seulement un intérêt temporel sans indiquer l'intérêt spatial avec $28,3 \%$, soit seulement un intérêt spatial sans aucune allusion à l'intérêt temporel avec $21,2 \%$.

Cette difficulté de mobilisation à la fois des facteurs temps et espace se manifeste encore plus nettement dans la description du déroulement du phénomène de fossilisation. En effet, 26,4 \% des lycéens ciblés n’ont fourni aucune réponse à la question $\mathrm{n}^{\circ} 3$ de notre questionnaire traitant de la relation fossile-encaissant. En outre, les explications données à cette question sont généralement ambiguës ou incomplètes et se répartissent entre les explications qui ne font allusion ni au facteur temps ni au facteur espace avec $61,4 \%$, en décrivant vaguement certains phénomènes géologiques comme la sédimentation, l'érosion, l'enfouissement ou la conservation des parties dures et les explications qui font appel au facteur temps avec 9,1\%, qui sont de trois types: celles signalant la succession temporelle de quelques étapes de la fossilisation sans préciser l'échelle de temps, celles ignorant totalement ces étapes mais mobilisant l'échelle Humaine et celles qualifiant le temps comme le seul facteur à l'origine des fossiles. Seules $3 \%$ des réponses ont mobilisé le facteur espace mais un espace figé puisqu'il est attribué à un "äbri" pour ces fossiles.

Ces réponses révèlent une méconnaissance du déroulement des processus de la fossilisation dans le temps et dans l'espace. On peut attribuer ce type de difficulté à l'incapacité de nos lycéens à concevoir un phénomène qui nécessite un temps long par rapport à l'échelle Humaine, et à imaginer des processus non constatés à l'œil nu, difficilement expérimentés et se déroulant dans des sites paléogéographiques différents.

Par ailleurs, si on examine le programme de géologie au secondaire qualifiant ou lycée, on constate qu'il consacre une bonne partie à l'étude des intérêts des fossiles sans faire référence ni aux chapitres concernant leur identification ni à ceux décrivant le déroulement du phénomène de fossilisation dans le temps et dans l'espace.

Ces différents aspects de difficultés de mobilisation des facteurs temps et espace peuvent aussi s'expliquer, comme souvent justifiés par les élèves, par des obstacles d'ordre pédagogique, en particulier le manque de travaux pratiques et de sorties sur le terrain. Ces derniers sont, en effet, 
essentielles pour développer la vision dans l'espace, le sens de l'orientation ainsi que les techniques de configuration bidimensionnelles et tridimensionnelles.

L'ensemble de nos résultats confirme l'hypothèse de notre recherche qui suppose que l'incapacité des élèves à mobiliser les facteurs temps et espace entrave leur compréhension du concept fossile.

Enfin, toutes ces difficultés d'assimilation des concepts géologiques peuvent être, en partie, à l'origine de la réticence des élèves vis-à-vis de la géologie tout au long de leur scolarité et probablement aussi dans leur orientation universitaire (Houari, 2015).

\section{Conclusion}

Nous avons réalisé ce travail afin d'identifier les difficultés et les obstacles relatifs à l'assimilation, par les lycéens marocains, des notions de temps et d'espace en géologie à travers l'étude du concept fossile.

Pour cela, nous avons dans un premier temps comparé le volume horaire alloué à la géologie avec celui consacré à la biologie dans les programmes des sciences de la vie et de la terre au secondaire. Le temps consacré à la géologie est de $33 \%$ au collège et de $27 \%$ au lycée par rapport à celui réservé à la biologie. Ceci influe négativement sur l'assimilation par les élèves des différents concepts et phénomènes géologiques enseignés.

Par la suite, nous avons mené une investigation basée sur la technique de groupe nominal et l'enquête par questionnaire, sur un échantillon de 135 lycéens marocains appartenant au lycée Moulay Rachid et au lycée Omo Ayman, de la ville de Fès. Les résultats obtenus nous ont permis de déceler quelques difficultés ressenties par les apprenants pour mobiliser les facteurs temps et espace lors de l'étude du concept fossile.

En effet, en définissant un fossile, ces apprenants ont rarement mobilisé les facteurs temps et espace simultanément. De plus, la minorité qui a fait intervenir ces deux facteurs survalorise le facteur temps au préjudice du facteur espace. Concernant l'intérêt spatio-temporel des fossiles, nos lycéens en sont conscients, mais l'intérêt temporel l'emporte toujours. Enfin, en expliquant la relation fossile-encaissant, ces élèves ont manifesté une grande difficulté à concevoir et/ou à imaginer la succession des étapes de la fossilisation dans le temps et dans l'espace. En outre, leurs explications se caractérisent souvent par une confusion dans la mobilisation des échelles de temps impliquées notamment l'échelle Humaine et l'échelle géologique.

En guise de suggestions, des mesures d'ordre pédagogique peuvent contribuer à l'atténuation de ces difficultés. Concernant les programmes, il serait primordial d'adapter le volume horaire alloué à la géologie au secondaire selon les objectifs fixés. Il faut aussi renforcer les équipements et les moyens pédagogiques nécessaires à une meilleure présentation des 
phénomènes géologiques, en particulier, des collections de fossiles diversifiées permettant de mieux illustrer la diversité des espèces fossiles animales et/ou végétales. Les sorties sur le terrain, quant à elles sont, essentielles pour développer la vision dans l'espace chez les apprenants.

Enfin pour nos perspectives de recherche, nous comptons élargir notre champ d'investigation aux étudiants des facultés des sciences inscrits en filière des sciences de la Terre et de l'Univers. Cela nous permettra de vérifier si ces étudiants, déjà entrain d'entamer leur spécialisation en géologie, continuent à rencontrer les mêmes types de difficultés, hérités du secondaire, vis-à-vis du concept fossile ou autres concepts de la discipline.

\section{References:}

1. Astolfi, J.-P. et Peterfalvi, B. (1993). Obstacles et construction de situations didactiques en sciences expérimentales. Recherches en didactique des sciences expérimentales(Aster), 16, 103-141. doi : $10.4267 / 2042 / 8578$

2. Boughanmi, Y. (2009). Obstacles à la problématisation du temps dans une approche Interdisciplinaire: l'explication de quelques phénomènes naturels par des lycéens et de futurs enseignants tunisiens (Thèse de doctorat). Université de Bourgogne et Université de Tunis, Bourgogne, France.

3. Chmanti-Houari, I., Oudrhiri Hassani M.et, Lachkhem, H. (2015). Causes de la réticence des étudiants marocains vis-à-vis de la géologie ; Cas de la faculté des sciences Dhar El Mehrez de Fès. Actes de la $1^{\mathrm{er}}$ Édition du Workshop International sur les Approches Pédagogiques et E-Learning.26-25 novembre 2015. Fès. Maroc.

4. Chalak, H. (2013). Magmatisme et conditions de construction de textes de savoirs problématisés au collège. Recherche en Education, HS, 5, 100-112. Récupéré du site de la revue: http : //www.recherches-en-education.net/IMG/pdf/REE-HS-5.pdf

5. Delbecq, A.L. and Van de Ven A.H. (1971). A group process model for problem identification and program planning. Journal of Applied Behavioral Science, 7(4), 466-492.

6. Encyclopédie des fossiles, édition Atlas 1987.

7. Gohau, G. (1990). Les sciences de la Terre aux XVIIe et XVIIIe siècles. Naissance de la géologie. Paris, France : Albin Michel.

8. Gohau, G. (1995). Traquer les obstacles épistémologiques à travers les lapsus d'élèves et d'écrivains. Recherches en didactique des sciences expérimentales(Aster), 20, 21-41. doi : 10.4267/2042/8624

9. Gouanelle, C. et Schneeberger, P. (1995). Enseigner les fossiles à l'école primaire. Recherches en didactique des sciences expérimentales(Aster), 21, 81-107. doi : 10.4267/2042/8637 
10. Grenier, J. et Lagarde, M. (2000). La technique du groupe nominale (TGN), une méthode de cueillette des données à connaître. In Renaissance et retombées de la recherche au collégial : actes du $12^{\mathrm{e}}$ colloque de l'Association pour la recherche au collégial (15-22). Collège de l'Outaouais Campus Félix-Leclerc, Québec.

11. Lamarti, L., Ben Bouziane, A., Akrim, H., Idrissi, M. et Talbi, M. (2009). L’hypermédia géo-terrain : un outil pertinent au service des apprentissages en géologie de terrain. Revue internationale des technologies en pédagogie universitaire, 6(1), 46-54.

12. Landry, R. (1993). L'analyse de contenu. In B. Gauthier (dir.), La recherche sociale (p. 337-359). Sainte-Foy, Québec: Presses de l’Université du Québec.

13. Laperrière-Tacussel, M. (1995). Le volcanisme, du cours moyen à L'IUFM. Recherches en didactique des sciences expérimentales (Aster), 20, 61-83. doi : 10.4267/2042/8626

14. Laperrière-Tacussel, M. (2002). Essai de construction de situations pédagogiques sur le thème des fossiles. Grand N, 69, 83-98.

15. Monchamp, A. et Sauvageot-Skibine, M. (1995). Du fixisme à la tectonique des plaques. Et pourtant elles bougent. Recherches en didactique des sciences expérimentales(Aster), 20, 3-20. doi : $10.4267 / 2042 / 8925$

16. Orange, D. (2003). Utilisation du temps et explications en sciences de la Terre par les élèves de lycée: étude dans quelques problèmes géologiques (Thèse de doctorat).Université de Nantes, France.

17. Savaton, P. (1998). La carte géologique dans l'enseignement secondaire. Bilan historique et didactique, réflexion et propositions d'apprentissage (Thèse de doctorat). Université de Paris VII -Denis Diderot, Paris, France. 